\title{
Functional Roles of the von Willebrand Factor Propeptide
}

\author{
Orla Rawley $^{1}$ David Lillicrap $^{1}$ \\ ${ }^{1}$ Department of Pathology and Molecular Medicine, Richardson \\ Laboratory, Queen's University, Kingston, Ontario, Canada \\ Hämostaseologie 2021;41:63-68.
}

\begin{abstract}
Address for correspondence David Lillicrap, MD, Department of Pathology and Molecular Medicine, Richardson Laboratory, Queen's University, Kingston, Ontario, K7L 3N6, Canada

(e-mail: david.lillicrap@queensu.ca).
\end{abstract}

\begin{abstract}
The primary polypeptide sequence of von Willebrand factor (VWF) includes an Nterminal 741-amino acid VWF propeptide (VWFpp). In cells expressing VWF, the VWFpp performs two critical functions. In the Golgi, VWFpp mediates the intermolecular disulfide linkages that generate high-molecular-weight VWF multimers. Subsequently, the VWFpp, which is proteolytically cleaved from mature VWF by furin, functions to generate the endothelial storage organelles (Weibel-Palade bodies) in which VWF and a distinct collection of proteins are stored, and from where they undergo regulated secretion from the endothelium. The VWFpp is secreted from endothelial cells as dimers and circulates in plasma with at least some of the dimers associating with a

Keywords

- von Willebrand disease

- von Willebrand factor

- propeptide

- VWFpp noncovalent manner with the D'D3 domain of mature VWF. The VWFpp has a half-life of 2 to 3 hours in plasma, but to date no extracellular function has been determined for the molecule. Nevertheless, its large size and several biologically interesting structural features (two sets of vicinal cysteines and an RGD sequence) suggest that there may be roles that the VWFpp plays in hemostasis or associated physiological processes such as angiogenesis or wound repair.
\end{abstract}

\section{Introduction}

von Willebrand factor (VWF) is a large adhesive glycoprotein that supports several key processes in the hemostatic response. The protein is synthesized by endothelial cells and megakaryocytes and some of the protein is stored in cytoplasmic organelles (Weibel-Palade bodies [WPBs] in the endothelium and $\alpha$ granules in platelets) from where it can be released upon appropriate stimulation.

The principal roles assigned to VWF in relation to hemostasis and thrombosis are its contributions to platelet plug formation, through binding to platelet glycoprotein Ib and through its carrier functions for the procoagulant cofactor, factor VIII (FVIII). ${ }^{1}$

This review will focus attention to an often-neglected component of VWF, its propeptide. The VWF propeptide (VWFpp), initially designated as VWFAgII, ${ }^{2,3}$ has a life cycle and functional role that show some similarities with the mature VWF protein but also has critical independent features that have been partially elucidated over the past 20 years. $^{4}$

\section{von Willebrand Factor Propeptide Structure}

von Willebrand factor is synthesized as large pre-pro-polypeptide sequence that undergoes extensive modification during its intracellular journey. Following transfer into the endoplasmic reticulum (ER), the N-terminal 22-amino acid signal peptide is released by proteolytic cleavage. The translated pro-VWF molecules undergo initial glycan additions and dimerization in the ER through disulfide bond generation at the C-terminus of the protein which then transfer to the Golgi where three further key posttranslational events occur ${ }^{5,6}$ : (1) further modification of the glycan chains, (2) the generation of very high-molecular-weight multimers of the VWF dimers, and (3) after facilitation of multimer generation, the cleavage received

November 2, 2020

accepted after revision

December 10, 2020
(C) 2021. Thieme. All rights reserved. Georg Thieme Verlag KG,

Rüdigerstraße 14,

70469 Stuttgart, Germany
DOI https://doi.org/ 10.1055/a-1334-8002. ISSN 0720-9355. 


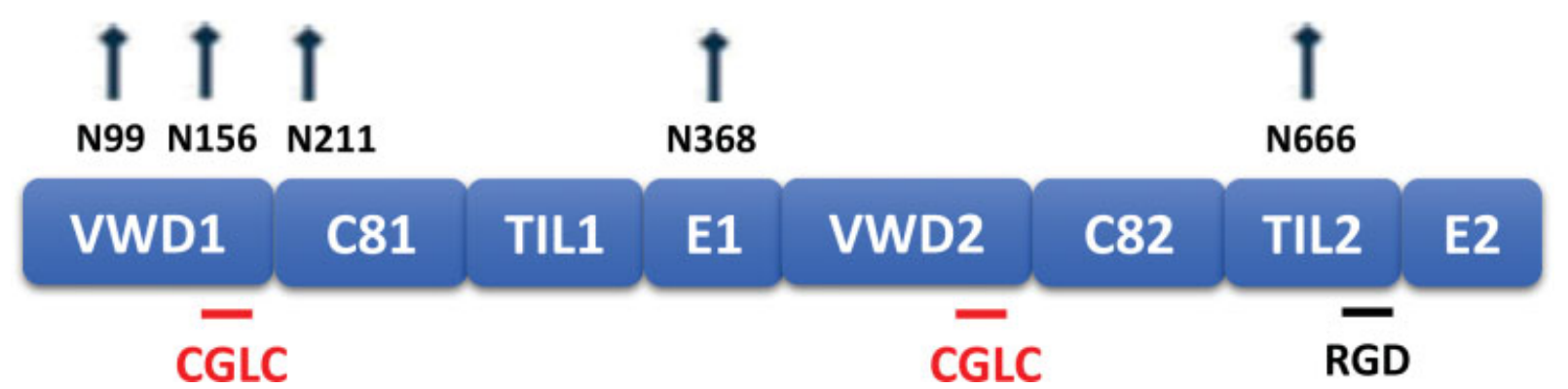

Fig. 1 Schematic representation of the von Willebrand factor propeptide (VWFpp) depicting the subdomain architecture of the D1 and D2 assemblies as well as key functional sites. The VWFpp is composed of two similar D assemblies each containing discrete functional sites. Each D assembly contains a vicinal disulfide in a "CGLC" motif as well as consensus sequences for N-linked glycosylation. VWFpp expresses canonical Asn$\mathrm{X}$-Ser/Thr glycosylation consensus sequences as well as noncanonical Asn-X-Cys sequences. In addition, the D2 assembly contains an integrinbinding "RGD" motif, the significance of which remains unclear.

of the 741 AA VWFpp from the mature VWF subunit (2050 AA in size) by the peptidase, furin. In the acidic environment of the Golgi (pH $\sim 6$ and $10 \mathrm{mM} \mathrm{Ca}^{2+}$ ), the newly released VWFpp forms a high affinity noncovalent association $\left(K_{\mathrm{D}}=0.2 \mathrm{nM}\right)$ with the $\mathrm{N}$-terminal $\mathrm{D}^{\prime} \mathrm{D} 3$ domain of the mature VWF subunit (-Fig. 1). ${ }^{\text {? }}$

Following processing in the Golgi, the nascent VWF multimers either undergo basal constitutive secretion from endothelial cells or are trafficked for storage in WPBs and $\alpha$-granules for regulated release from the endothelium and platelets, respectively. In blood, the newly released VWF multimers undergo limited proteolysis mediated by the metalloprotease ADAMTS13 (a disintegrin and metalloproteinase with a thrombospondin type 1 motif, member 13), while dimers of the cleaved VWFpp are released in equimolar quantities, and some of these dimers circulate in a noncovalent association with the mature VWF multimers. In the plasma environment (pH 7.4 and $2.5 \mathrm{mMCa}^{2+}$ ), this association with the mature VWF $\mathrm{D}^{\prime} \mathrm{D} 3$ domain remains of high affinity $K_{\mathrm{D}} \sim 50 \mathrm{nM}$. $^{7}$ The precise ratio of VWF-associated versus free VWFpp dimers in the circulation has not been established.

The 741-AAVWFpp possesses several structural details that prove to be crucial for VWF expression and distinct aspects of VWF function. The full-length VWF polypeptide possesses 16 $\mathrm{N}$-linked glycosylation sites, 4 of which are located in the VWFpp. ${ }^{8}$ In addition to the four consensus Asn-X-Ser/Thr glycan sequons at Asn 99, Asn 156, Asn 211, and Asn 666, there are two additional noncanonical $\mathrm{N}$-glycan attachment sites at Asn-X-Cys (Asn 368 and Asn 663) that are at least partially occupied. Studies of these glycosylation sites in the VWFpp indicate that mutation of the initial Asn 99 residue dramatically interrupts VWF expression, with the mutant protein being sequestered in the ER. ${ }^{9}$ The functional role of the remaining VWFpp glycans remains to be further studied, but preliminary in vitro investigations suggest that the glycan additions at Asn 156 and Asn 666 play an important early role in facilitating platelet adhesion under arterial shear to a collagen surface.

Another early discovery with regard to VWFpp structure/ function is its role in mediating multimer formation through the generation of intermolecular disulfide linkages at the $\mathrm{N}$ terminus of VWF dimers. ${ }^{5,10}$ This critical event occurs in the acidic environment of the Golgi and requires the disulfide isomerase activity of two sets of vicinal cysteines in the D1 (AAs 159-162) and D2 domains (AAs 521-524) of the VWFpp. ${ }^{11,12}$ Mutagenesis of either of these structures significantly disrupts multimer formation. In contrast, while physiological multimer assembly is mediated by the VWFpp in cis with the mature VWF subunit, early studies demonstrated that this event can also proceed with the VWFpp present in trans, although whether this observation has any physiological relevance is unclear. ${ }^{13}$

The other noteworthy structural feature of the VWFpp is an RGD motif in the TIL-2 region of the D2 domain. This structural element suggests that the VWFpp may participate in an integrin interaction, but evidence for this activity and its potential physiological relevance have yet to be explored.

\section{Pathogenic VWFpp Variants}

Approximately 150 unique coding and noncoding variants have been documented within the region of the VWF gene responsible for encoding the VWFpp. ${ }^{14}$ The pathobiological significance of many of these variants has not yet been established and further investigation is required to determine whether or not these variants are indeed pathogenic. However, for several exon 1-17 coding variants, the pathogenic significance is clear. For example, VWFpp variants p.Tyr87Ser and p. Cys570Ser cause a distinct subtype of recessive type $2 \mathrm{~A}$ von Willebrand disease (VWD). ${ }^{15,16}$ These variants negatively impact VWF multimerization such that dimeric VWF alone is detectable in the plasma of patients. In addition to multimerization defects, loss of regulated storage and secretory defects have also been described for several type $2 \mathrm{~A}$ causing VWFpp variants including the p.Asn528Ser variant. ${ }^{17}$ Furthermore, VWFpp variants have been associated with both type 1 and type 3 VWD. ${ }^{18-21}$

\section{Intracellular Function of the VWF Propeptide}

As alluded to above, the best characterized functional role of the VWFpp is its essential role in facilitating the formation of VWF multimer assembly, a process requiring the two sets of vicinal cysteines in the D1 and D2 domains. ${ }^{11}$ The disulfide isomerase activity mediated by these structures is required to generate the intermolecular disulfide bonds between $\mathrm{N}$-terminal $\mathrm{D}^{\prime} \mathrm{D} 3$ 




Fig. 2 Intracellular and extracellular functions of the von Willebrand factor propeptide (VWFpp). VWFpp mediates VWF multimerization. The primary role of the VWFpp is directing VWF multimerization. Multimerization involves a unique mechanism that requires the endogenous oxidoreductase activity of the two vicinal disulfides (CGLC motifs) of the VWFpp. ProVWF dimers are disulfide linked to form higher-order multimers through the formation of a transient intracellular disulfide bond between the VWFpp and the D'D3 domains of VWF. Once multimerization is complete, VWFpp is cleaved by furin, and is stored as a dimer along with mature VWF in the WPBs. Potential role for VWFpp in hemostasis. Although VWFpp is cosecreted with VWF from WPBs, a definitive hemostatic role for VWFpp has not been described. However, given its relative abundance at sites of vascular injury, potential functions may include anchoring VWF at the endothelial cell surface, tethering platelets, or acting as a molecular bridge connecting adjacent VWF molecules to reinforce the hemostatic plug. VWFpp clearance. Following secretion, VWFpp circulates in the plasma with a half-life of $\sim 2$ hours. However, the mechanisms regulating VWFpp clearance remain poorly understood. To date, studies have demonstrated that the scavenger receptor, stabilin-2, which is expressed on the liver sinusoidal endothelium, is a major regulator of VWFpp clearance. There also appears to be a lesser contribution from the scavenger receptor SCARA5, and the contribution of other clearance receptors such as CLEC4M is an area of active investigation.

domains of the mature subunit dimers. This event occurs in the acidic environment of the Golgi, and once completed the VWFpp is cleaved from the newly generated multimers by furin at the paired basic amino acid sequence between residues 741 and 742 (-Fig. 2).

The second intracellular function requiring VWFpp is the development of its cytoplasmic storage organelles. ${ }^{22}$ This process has been examined in a detailed fashion for WPBs in endothelial cells. ${ }^{23}$ These studies have shown that sequences in the D2 domain of the VWFpp mediate WPB formation and that WPB storage of proteins that are not routinely targeted to these organelles can be accomplished by expressing them as fusion partners with the VWFpp. ${ }^{24,25}$ Of note, analysis of the role of the VWFpp in the generation of $\alpha$ granules in megakaryocytes/platelets, where the shape and size of organelle are distinct, suggests a different structural basis for this process. ${ }^{26}$

\section{Extracellular Function of the VWF Propeptide}

There are several features of the VWFpp that suggest an extracellular role for the protein. The VWFpp is much larger than most propeptide sequences and once secreted from its cell of synthesis it circulates with a half-life of 2 to 3 hours 4 before being cleared by mechanisms that are still being investigated. VWFpp possesses several structural motifs (especially the D2 domain RGD sequence) that might have functional relevance in hemostasis or related biological processes and, lastly, documentation of the clinical bleeding phenotype in type 3 VWD patients has shown significantly higher bleeding scores in those patients with mutations in the VWFpp sequence. ${ }^{21}$

The limited investigation of potential extracellular functions of the VWFpp has to date resulted in discordant results using reagents that also differ in their species derivation. A series of studies utilizing bovine VWFpp have shown binding to bovine type 1 collagen and to the very late antigen- 4 integrin, and also demonstrated that the bovine VWFpp could be cross-linked by FXIIIa to laminin. ${ }^{27}$ More recent studies utilizing human VWFpp have shown an interference with VWF binding to platelets, thus reducing platelet activation and adhesion. ${ }^{7}$ Furthermore, human VWFpp binding to equine collagen was not detected under either static or microfluidic conditions.

While the VWFpp exhibits well-documented disulfide isomerase activity in the acidic environment of the Golgi to mediate the intermolecular disulfide linkages required for VWF multimer assembly, at pH 7.4 in plasma there is no evidence that a similar function is active (O. Rawley, VWFpp function, personal communication, 2020). 
Most recently, we have examined the role of the highly conserved murine VWFpp in supporting platelet adhesion to a collagen surface under arterial flow conditions $\left(2,500 \mathrm{~s}^{-1}\right)$. These studies have demonstrated a supplementary role for the VWFpp during the early stages of platelet adhesion and have also shown that this function requires glycan additions at Asn residues 156 and 666 (0. Rawley, personal communication, 2020). A more detailed analysis of the imaging results from these studies shows that the effect of the coexistent VWFpp is to enhance the mass of the platelet thrombus that develops. Further studies addressing the potential of VWFpp's facilitation of the initial hemostatic process are now ongoing in murine in vivo models of hemostasis and thrombosis.

For the various reasons listed previously, it seems logical that the VWFpp should have some distinct extracellular function following its release from its cell of synthesis. Whether this is associated with similar hemostatic functions to the mature VWF multimers or has an independent role in a related "repair" process such as angiogenesis or wound healing remains to be explored.

\section{Clearance of the VWF Propeptide and Its Role as Biomarker}

While the biosynthesis of the VWFpp has been well documented and its presence in the plasma also well characterized, much less has been known about the clearance of VWFpp from the circulation. In studies focused on murine VWFpp, we have now shown that the sinusoidal scavenger receptor, stabilin-2, that contributes significantly to the clearance of mature VWF, also plays an important role in VWFpp clearance. ${ }^{28}$ Infusion of VWFpp into VWF/Stab2 double knockout mice prolongs the half-life of VWFpp fourfold compared with VWF single knockout mice and the baseline plasma levels of VWFpp in Stab2 knockout mice are significantly elevated $(p<0.0001)$ compared with WT animals. We have also recently documented a contribution to mouse VWFpp clearance by another scavenger receptor, SCARA5, although the magnitude of this effect appears to be minor. ${ }^{29}$ Importantly, and as highlighted in other parts of this review, interspecies conclusions should be made with caution, and despite the high level of homology between mouse and human VWFpp AA sequences (86\%), plasma levels of VWFpp are not elevated in humans known to be heterozygous for loss-of-function stabilin-2 variants. ${ }^{30}$

While we are only very recently learning about the details of how and where the VWFpp is cleared from the circulation, measurement of VWFpp levels in plasma has been used for several years as a biomarker of certain pathophysiologic states. Concomitant increases in VWFpp and mature VWF: $\mathrm{Ag}$ are found in situations of acute endothelial activation such as thrombotic thrombocytopenic purpura, hemolytic uremic syndrome, sepsis, and severe malaria. ${ }^{31}$ In contrast, in conditions in which endothelial cell pathology is more chronic in nature, only VWF is significantly elevated and not VWFpp. Coincident elevations of VWFpp and VWF can also appear with platelet activation, either as a preanalytical variable due to blood sampling or processing artifacts, or as a marker of pathological platelet activation.

The other instance in which VWFpp measurement has gained acceptance for clinical purposes concerns the use of the VWFpp/VWF:Ag ratio as a surrogate for VWF clearance assessment. In healthy subjects, this ratio will be less than 3 , whereas in VWD patients with accelerated clearance of VWF (in whom VWFpp clearance will be unchanged) the VWFpp/VWF:Ag ratio will be increased, sometimes to greater than $10 .^{32,33}$ This VWF laboratory phenotype is a marker of type $1 \mathrm{C}$ (clearance) VWD, of which the archetype example is the VWD Vicenza (p.Arg1205His) variant. In these patients, the plasma VWF levels are usually 0.10 to $0.15 \mathrm{IU} / \mathrm{mL}$, and although they usually respond very well to desmopressin administration, with excellent VWF release from the endothelium (5- to 10 -fold increments of VWF), the response is transient, with a return to baseline levels by 4 hours post-administration. This response reflects the markedly reduced half-life of the multimers containing the variant VWF (the phenotype manifests in heterozygotes) that can be as much as 10 -fold shorter than normal. Thus, in type 1C VWD patients, desmopressin may be used for satisfactory hemostatic control for minor interventions but not for major procedures.

Incorporation of VWFpp measurement has also been proposed as an adjunctive test to classify other VWD subtypes, and has been especially useful in differentiating severe type 1 from type 3 disease. ${ }^{34,35}$ VWFpp measurement is routinely achieved via the enzyme-linked immunosorbent assay (ELISA) format. Currently, however, there is little consensus on the specific advantages of choosing one particular VWFpp ELISA over another. Both commercially available and in-house VWFpp ELISAs are currently utilized in the diagnostic setting, with little evidence of differential performance. ${ }^{36}$

\section{Therapeutic Considerations and the VWF Propeptide}

When therapeutic interventions are required for VWD patients, they are usually prompted by a requirement to prevent or treat bleeding. These interventions can be categorized into two classes of agents-non-VWF hemostatic compounds and VWF products.

The list of non-VWF agents that enhance hemostasis in patients with deficient or defective VWF includes estrogenic hormones, antifibrinolytic drugs, and desmopressin. ${ }^{37}$ of this list, we are only aware of changes to VWFpp induced by desmopressin administration, following which there will be a release of VWFpp that is equivalent to that of the mature VWF molecule. One might assume that estrogens, which increase the plasma levels of VWF, also result in higher plasma VWFpp levels, but this assumption has not been verified.

The other principal mode of treatment for defects of VWF involves infusion of VWF concentrates derived from either plasma donations or, more recently, recombinant DNA technology. ${ }^{38}$ Extensive clinical evaluation of the plasma-derived VWF concentrates available around the world indicates that they are safe and generally very effective for preventing or 
treating bleeding where there is a deficiency or functional defect of VWF. Only in certain clinical circumstances such as recurrent gastrointestinal bleeding related to angiodysplasia are there treatment failures with the appropriate dosing and administration schedule of VWF concentrates. ${ }^{38}$

There have been claims that certain VWF concentrates may provide enhanced hemostasis due to the presence of higher-molecular-weight forms of VWF and this could certainly be a valid possibility for the recombinant VWF product that has not been modified by prior exposure to ADAMTS13 at the time of infusion. However, to date, although these claims have biological plausibility, there is only anecdotal evidence to support the proposal that any one concentrate is more clinically efficacious than another. Another related issue to be kept in mind is that the VWFpp is coinfused with the various plasma-derived VWF concentrates but is virtually absent from the recombinant VWF product. If the VWFpp does indeed have a supplementary hemostatic or related biological role, this might be missing in treatments involving the recombinant VWF product, although clinical trials of this concentrate in a range of clinical contexts have shown no evidence of reduced efficacy. ${ }^{39,40}$

\section{Conclusion}

Von Willebrand factor is a critical contributor to the body's hemostatic response, and pathological conditions involving VWF are well documented to result in bleeding and thrombotic outcomes. However, almost all of this knowledge has focused on the functions associated with the mature VWF subunit. While it is clear that the VWFpp plays a crucial role in facilitating VWF multimer assembly in the Golgi, and in the generation of and trafficking to WPBs, the fact that it is secreted and circulates for 2 hours without an apparent extracellular function remains mysterious and merits further investigation.

\section{Funding}

D.L. is the recipient of a Canada Research Chair in Molecular Hemostasis. The authors' research concerning the VWFpp is funded in part by the Canadian Institutes of Health Research Foundation Grant (FDN154285).

\section{Conflict of Interest}

The authors declare that they have no conflict of interest.

\section{References}

1 Ruggeri ZM. The role of von Willebrand factor in thrombus formation. Thromb Res 2007;120(01, Suppl 1):S5-S9

2 Fay PJ, Kawai Y, Wagner DD, et al. Propolypeptide of von Willebrand factor circulates in blood and is identical to von Willebrand antigen II. Science 1986;232(4753):995-998

3 Kawai Y, Montgomery RR. Endothelial cell processing of von Willebrand proteins. Ann N Y Acad Sci 1987;509:60-70

4 Haberichter SL. von Willebrand factor propeptide: biology and clinical utility. Blood 2015;126(15):1753-1761

5 Sadler JE. von Willebrand factor assembly and secretion. J Thromb Haemost 2009;7(Suppl 1):24-27
6 Lenting PJ, Christophe OD, Denis CV. von Willebrand factor biosynthesis, secretion, and clearance: connecting the far ends. Blood 2015;125(13):2019-2028

7 Madabhushi SR, Shang C, Dayananda KM, et al. von Willebrand factor (VWF) propeptide binding to VWF D'D3 domain attenuates platelet activation and adhesion. Blood 2012;119(20):4769-4778

8 Canis K, McKinnon TAJ, Nowak A, et al. Mapping the N-glycome of human von Willebrand factor. Biochem J 2012;447(02):217-228

9 McKinnon TAJ, Goode EC, Birdsey GM, et al. Specific N-linked glycosylation sites modulate synthesis and secretion of von Willebrand factor. Blood 2010;116(04):640-648

10 Verweij CL, Hart M, Pannekoek H. Expression of variant von Willebrand factor ( $v W F$ ) CDNA in heterologous cells: requirement of the pro-polypeptide in vWF multimer formation. EMBO J 1987; 6(10):2885-2890

11 Mayadas TN, Wagner DD. Vicinal cysteines in the prosequence play a role in von Willebrand factor multimer assembly. Proc Natl Acad Sci U S A 1992;89(08):3531-3535

12 Purvis AR, Sadler JE. A covalent oxidoreductase intermediate in propeptide-dependent von Willebrand factor multimerization. J Biol Chem 2004;279(48):49982-49988

13 Wise RJ, Pittman DD, Handin RI, Kaufman RJ, Orkin SH. The propeptide of von Willebrand factor independently mediates the assembly of von Willebrand multimers. Cell 1988;52(02):229-236

14 Hampshire DJ, Goodeve AC. The international society on thrombosis and haematosis von Willebrand disease database: an update. Semin Thromb Hemost 2011;37(05):470-479

15 Rosenberg JB, Haberichter SL, Jozwiak MA, et al. The role of the D1 domain of the von Willebrand factor propeptide in multimerization of VWF. Blood 2002;100(05):1699-1706

16 Lanke E, Kristoffersson AC, Philips M, Holmberg L, Lethagen S. Characterization of a novel mutation in the von Willebrand factor propeptide in a distinct subtype of recessive von Willebrand disease. Thromb Haemost 2008;100(02):211-216

17 Haberichter SL, Budde U, Obser T, Schneppenheim S, Wermes C, Schneppenheim R. The mutation N528S in the von Willebrand factor (VWF) propeptide causes defective multimerization and storage of VWF. Blood 2010;115(22):4580-4587

18 James PD, Notley C, Hegadorn C, et al. The mutational spectrum of type 1 von Willebrand disease: results from a Canadian cohort study. Blood 2007;109(01):145-154

19 Goodeve A, Eikenboom J, Castaman G, et al. Phenotype and genotype of a cohort of families historically diagnosed with type 1 von Willebrand disease in the European study, Molecular and Clinical Markers for the Diagnosis and Management of Type 1 von Willebrand Disease (MCMDM-1VWD). Blood 2007;109(01): 112-121

20 Baronciani L, Federici AB, Cozzi G, et al. Expression studies of missense mutations p.D141Y, p.C275S located in the propeptide of von Willebrand factor in patients with type 3 von Willebrand disease. Haemophilia 2008;14(03):549-555

21 Bowman M, Tuttle A, Notley C, et al;Association of Hemophilia Clinic Directors of Canada. The genetics of Canadian type 3 von Willebrand disease: further evidence for co-dominant inheritance of mutant alleles. J Thromb Haemost 2013;11(03):512-520

22 Wagner DD, Saffaripour S, Bonfanti R, et al. Induction of specific storage organelles by von Willebrand factor propolypeptide. Cell 1991;64(02):403-413

23 Mourik M, Eikenboom J. Lifecycle of Weibel-Palade bodies. Hamostaseologie 2017;37(01):13-24

24 Haberichter SL, Jozwiak MA, Rosenberg JB, Christopherson PA, Montgomery RR. The von Willebrand factor propeptide (VWFpp) traffics an unrelated protein to storage. Arterioscler Thromb Vasc Biol 2002;22(06):921-926

25 Haberichter SL, Jacobi P, Montgomery RR. Critical independent regions in the VWF propeptide and mature VWF that enable normal VWF storage. Blood 2003;101(04):1384-1391 
26 Chen CH, Lo RW, Urban D, Pluthero FG, Kahr WHA. $\alpha$-Granule biogenesis: from disease to discovery. Platelets 2017;28(02): 147-154

27 Takagi J, Fujisawa T, Sekiya F, Saito Y. Collagen-binding domain within bovine propolypeptide of von Willebrand factor. J Biol Chem 1991;266(09):5575-5579

28 Rawley O, Brown C, Dwyer C, Nesbitt K, Lillicrap D. Clearance of mouse VWF propeptide is mediated by stabilin-2 and is regulated by N-linked glycan expression. Res Pract Thromb Haemost 2018;2 (S1):1-368

29 Rawley O, Nesbitt K, Dwyer C, Swystun L, Lillicrap D. The scavenger receptor SCARA5 mediates clearance of the von Willebrand factor propeptide. Res Pract Thromb Haemost 2020;4(S2):1-1311

30 Desch KC, Ozel AB, Halvorsen M, et al. Whole-exome sequencing identifies rare variants in STAB2 associated with venous thromboembolic disease. Blood 2020;136(05):533-541

31 van Mourik JA, Romani de Wit T. Von Willebrand factor propeptide in vascular disorders. Thromb Haemost 2001;86(01):164-171

32 Haberichter SL, Castaman G, Budde U, et al. Identification of type 1 von Willebrand disease patients with reduced von Willebrand factor survival by assay of the VWF propeptide in the European study: molecular and clinical markers for the diagnosis and management of type 1 VWD (MCMDM-1VWD). Blood 2008;111 (10):4979-4985
33 Haberichter SL, Balistreri M, Christopherson P, et al. Assay of the von Willebrand factor (VWF) propeptide to identify patients with type 1 von Willebrand disease with decreased VWF survival. Blood 2006;108(10):3344-3351

34 Sanders YV, Groeneveld D, Meijer K, et al;WiN Study Group. von Willebrand factor propeptide and the phenotypic classification of von Willebrand disease. Blood 2015;125(19):3006-3013

35 Haberichter SL. VWF propeptide in defining VWD subtypes. Blood 2015;125(19):2882-2883

36 Hubbard AR, Hamill M, Eikenboom HCJ, Montgomery RR, Mertens $\mathrm{K}$, Haberichter SSSC Sub-Committee on von Willebrand Factor of ISTH. Standardization of von Willebrand factor propeptide: value assignment to the WHO 6th IS Factor VIII/von Willebrand factor, plasma (07/316). J Thromb Haemost 2012;10(05):959-960

37 Sharma R, Flood VH. Advances in the diagnosis and treatment of Von Willebrand disease. Blood 2017;130(22):2386-2391

38 Mannucci PM. New therapies for von Willebrand disease. Blood Adv 2019;3(21):3481-3487

39 Gill JC, Castaman G, Windyga J, et al. Hemostatic efficacy, safety, and pharmacokinetics of a recombinant von Willebrand factor in severe von Willebrand disease. Blood 2015;126(17):2038-2046

40 Peyvandi F, Mamaev A, Wang JD, et al. Phase 3 study of recombinant von Willebrand factor in patients with severe von Willebrand disease who are undergoing elective surgery. J Thromb Haemost 2019;17(01):52-62 